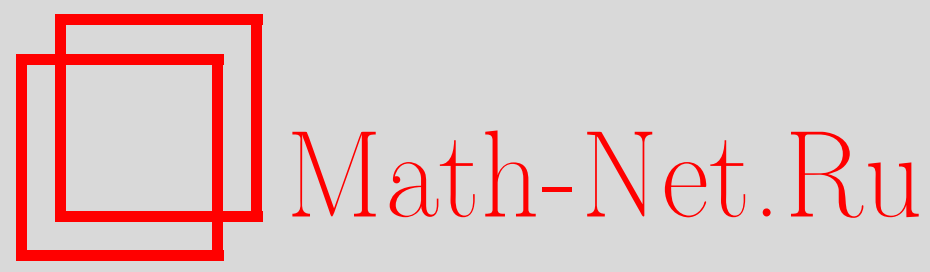

А. С. Романюк, Приближение классов периодических функций многих переменных, Матем. заметки, 2002, том 71, выпуск 1, 109-121

DOI: https://doi.org/10.4213/mzm332

Использование Общероссийского математического портала Math-Net.Ru подразумевает, что вы прочитали и согласны с пользовательским соглашением http://www.mathnet.ru/rus/agreement

Параметры загрузки:

IP : 3.89 .197 .203

26 апреля 2023 г., 15:02:35

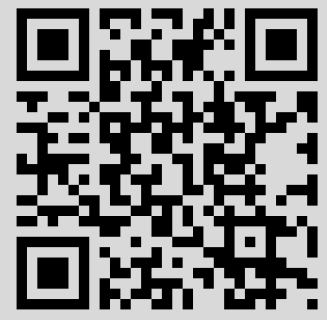




\title{
ПРИБЛИЖЕНИЕ КЛАССОВ ПЕРИОДИЧЕСКИХ ФУНКЦИЙ МНОГИХ ПЕРЕМЕННЫХ
}

\section{А. С. Романюк}

\begin{abstract}
Изучается приближение классов периодических функций многих переменных $B_{p, \theta}^{r}$ и $W_{p, \alpha}^{r}$ кратными суммами Фурье фиксированного порядка, построенньми с учетом индивидуальных свойств функций из этих классов. Такой выбор аппроксимирующих агрегатов в ряде случаев позволяет добиться лучшего порядка приближения указанных классов по сравнению с их приближением ступенчатьми гиперболическими суммами Фурье.

Библиография: 10 названий.
\end{abstract}

В работе рассматриваются так называемые ортогональные тригонометрические приближения классов периодических функций многих переменных $B_{p, \theta}^{r}$ и $W_{p, \alpha}^{r}$ в пространстве $L_{q}$. Величины, о которых идет речь, являются частным случаем наилучших $M$ членных тригонометрических приближений (см. [1], [2]), которые особенно интенсивно исследуются в последнее время.

Приведем необходимые в дальнейшем обозначения и определения.

Пусть $\mathbb{R}^{d}, d \geqslant 1,-$ евклидово пространство с элементами $x=\left(x_{1}, \ldots, x_{d}\right)$ и $(x, y)=$ $x_{1} y_{1}+\cdots+x_{d} y_{d} ; L_{p}\left(\pi_{d}\right), \pi_{d}=\prod_{j=1}^{d}[-\pi ; \pi]$, обозначает множество функций $f(x)$, $2 \pi$-периодических по каждой переменной, таких, что

$$
\begin{aligned}
\|f\|_{p}= & \left((2 \pi)^{-d} \int_{\pi_{d}}|f(x)|^{p} d x\right)^{1 / p}<\infty, \quad 1 \leqslant p<\infty, \\
& \|f\|_{\infty}=\underset{x \in \pi_{d}}{\operatorname{vrai} \sup _{d}}|f(x)|<\infty, \quad p=\infty .
\end{aligned}
$$

Всюду ниже предполагаем, что для $f(x) \in L_{p}\left(\pi_{d}\right)$ вьполнено условие

$$
\int_{-\pi}^{\pi} f(x) d x_{j}=0, \quad j=1, \ldots, d .
$$

Для векторов $k=\left(k_{1}, \ldots, k_{d}\right), k_{j} \in \mathbb{Z}$, и $s=\left(s_{1}, \ldots, s_{d}\right), s_{j} \in \mathbb{N}, j=1, \ldots, d$, положим $\rho(s)=\left\{k: 2^{s_{j}-1} \leqslant\left|k_{j}\right|<2^{s_{j}}\right\}$ и для $f(x) \in L_{p}\left(\pi_{d}\right)$ обозначим

$$
\delta_{s}(f, x)=\sum_{k \in \rho(s)} \widehat{f}(k) e^{i(k, x)},
$$

где $\widehat{f}(k)=(2 \pi)^{-d} \int_{\pi_{d}} f(t) e^{-i(k, t)} d t-$ коэффициенты Фурье $f(x)$. 
Пусть $1<p<\infty, r=\left(r_{1}, \ldots, r_{d}\right), r_{j}>0, j=1, \ldots, d$. Тогда классы Бесова $B_{p, \theta}^{r}$ определяются следующим образом (см., например, [3]):

$$
B_{p, \theta}^{r}=\left\{f(x) \mid\|f\|_{B_{p, \theta}^{r}}=\left(\sum_{s} 2^{(s, r) \theta}\left\|\delta_{s}(f, x)\right\|_{p}^{\theta}\right)^{1 / \theta} \leqslant 1\right\}
$$

при $1 \leqslant \theta<\infty$ и

$$
B_{p, \infty}^{r}=\left\{f(x) \mid\|f\|_{B_{p, \infty}^{r}}=\sup _{s} 2^{(s, r)}\left\|\delta_{s}(f, x)\right\|_{p} \leqslant 1\right\} .
$$

Напомним, что классы $B_{p, \infty}^{r}$ совпадают с классами $H_{p}^{r}$ (см. $[1$, с. 31]), которые являются аналогами известных классов Никольского (см. [4, с. 189]). Параллельно нами будут исследоваться также классы $W_{p, \alpha}^{r}$. Напомним их определение (см., например, [1, с. 31]).

Пусть $F_{r}(x, \alpha)$ обозначают многомерные аналоги ядер Бернулли, т.е.

$$
F_{r}(x, \alpha)=2^{d} \sum_{k} \prod_{j=1}^{d} k_{j}^{-r_{j}} \cos \left(k_{j} x_{j}-\frac{\alpha_{j} \pi}{2}\right), \quad r_{j}>0, \quad \alpha_{j} \in \mathbb{R},
$$

и в сумме участвуют только те векторы $k$, для которых $k_{j}>0, j=1, \ldots, d$. Обозначим $W_{p, \alpha}^{r}$ класс функций $f(x)$, представимых в виде

$$
f(x)=\varphi(x) * F_{r}(x, \alpha)=(2 \pi)^{-d} \int_{\pi_{d}} \varphi(y) F_{r}(x-y, \alpha) d y,
$$

где $\varphi(x) \in L_{p}\left(\pi_{d}\right),\|\varphi\|_{p} \leqslant 1$.

В дальнейшем, не умаляя общности, будем считать, что координаты вектора $r=$ $\left(r_{1}, \ldots, r_{d}\right)$ упорядочены в виде: $0<r_{1}=r_{2}=\cdots=r_{\nu}<r_{\nu+1} \leqslant \cdots \leqslant r_{d}$. Вектору $r=\left(r_{1}, \ldots, r_{d}\right)$ будем сопоставлять вектор $\gamma=\left(\gamma_{1}, \ldots, \gamma_{d}\right), \gamma_{j}=r_{j} / r_{1}, j=1, \ldots, d$, которому, в свою очередь, сопоставляется вектор $\gamma^{\prime}=\left(\gamma_{1}^{\prime}, \ldots, \gamma_{d}^{\prime}\right)$, где $\gamma_{j}=\gamma_{j}^{\prime}$ при $j=1, \ldots, \nu$ и $1<\gamma_{j}^{\prime}<\gamma_{j}, j=\nu+1, \ldots, d$. Если $A$ - конечное множество, то через $|A|$ будем обозначать количество его элементов.

Пусть $\Omega_{M}$ - произвольный набор из $M d$-мерных векторов $k^{1}, \ldots, k^{M}$ с целочисленными координатами и $T\left(\Omega_{M}, x\right)$ - подпространство тригонометрических полиномов, порожденное системой экспонент $\left\{e^{i\left(k^{j}, x\right)}\right\}_{j=1}^{M}$. Полагая для $f(x) \in L_{p}\left(\pi_{d}\right) S_{M}(f, x)=$ $P\left(f, T\left(\Omega_{M}, x\right)\right)$, где $P\left(f, T\left(\Omega_{M}, x\right)\right)$ - ортогональная проекция $f(x)$ на $T\left(\Omega_{M}, x\right)$, pacсмотрим величину

$$
e_{M}^{\perp}(f)_{q}=\inf _{\left\{k^{j}\right\}}\left\|f(x)-S_{M}(f, x)\right\|_{q} .
$$

Если $K \subset L_{p}\left(\pi_{d}\right)$ - некоторьй класс функций, то полагаем

$$
e_{M}^{\perp}(K)_{q}=\sup _{f \in K} e_{M}^{\perp}(f)_{q} .
$$

Отметим, что в том случае, когда $K=W_{p, \alpha}^{r}$, величины (1) для некоторых соотношений между параметрами $p$ и $q$ исследовались в [5].

Все приводимые ниже результаты будем формулировать в терминах порядковых соотношений. При этом функции $\mu_{1}(\mathbb{N})$ и $\mu_{2}(\mathbb{N})$ назьваем функииями одного порядка и пишем $\mu_{1} \asymp \mu_{2}$, если сушествуют постоянные $C_{1}$ и $C_{2}$ такие, что $C_{1} \mu_{2}(\mathbb{N}) \leqslant \mu_{1}(\mathbb{N}) \leqslant$ $C_{2} \mu_{2}(\mathbb{N})$. Аналогичным образом определяются порядковые неравенства $\mu_{1} \ll \mu_{2}$ и $\mu_{1} \gg \mu_{2}$. Отметим, что ниже постоянные $C_{i}, i=1,2, \ldots$, могут зависеть только от параметров, определяюших классы, метрики $L_{q}$ и размерности пространства $\mathbb{R}^{d}$.

Теперь перейдем к изложению полученных результатов. 
Tеорема 1. Пусть $1<p<q<\infty, r_{1}>1 / p-1 / q$. Тогда прu $1 \leqslant \theta \leqslant \infty$ имеет место порядковое соотношение

$$
e_{M}^{\perp}\left(B_{p, \theta}^{r}\right)_{q} \asymp M^{-\left(r_{1}-1 / p+1 / q\right)}\left(\log ^{\nu-1} M\right)^{\left(r_{1}-1 / p+2 / q-1 / \theta\right)_{+}},
$$

əде $a_{+}=\max \{a, 0\}$.

ДокАЗАТЕЛьство. Получим сначала в (2) оценку сверху. Предварительно заметим, что в случае $\theta \geqslant q$ требуемая оценка сверху следует из соответствующей оценки приближения класса $B_{p, \theta}^{r}$ ступенчатьми гиперболическими суммами Фурье

$$
S_{n}^{\gamma}(f, x)=\sum_{(s, \gamma)<n} \delta_{s}(f, x)
$$

(см. [6, теорема 2]), если $M$ и $n$ связаны соотношением $M \asymp 2^{n} n^{\nu-1}$. Таким образом, ниже при установлении оценки сверху будем предполагать, что $\theta \in[1, q)$.

Итак, пусть $f(x) \in B_{p, \theta}^{r}, 1 \leqslant \theta<q$. По числу $M$ подберем $n \in \mathbb{N}$ из соотношения $M \asymp 2^{n} n^{\nu-1}$ и положим $n_{0}=[n+(\nu-1) \log n]$, где $[a]-$ целая часть числа $a$. Полином $S_{M}(f, x)$, доставляющий для $f(x) \in B_{p, \theta}^{r}$ требуемую оценку приближения, будем подбирать в виде

$$
S_{M}(f, x)=\sum_{(s, \gamma)<n} \delta_{s}(f, x)+R(x),
$$

где $R(x)$ - некоторьй полином, к построению которого мы и перейдем.

Каждому натуральному числу $l, n \leqslant l<n_{0}$, сопоставим величину

$$
S_{l}=\left(\sum_{l \leqslant(s, \gamma)<l+1} 2^{(s, r) \theta}\left\|\delta_{s}(f, x)\right\|_{p}^{\theta}\right)^{1 / \theta}
$$

и положим

$$
m_{l}=\left[2^{n} n^{\nu-1} 2^{-l} S_{l}^{\theta}\right]+1
$$

Далее, пусть $\alpha_{i}(f, l), i=1,2, \ldots$, обозначают числа $\left\|\delta_{s}(f, x)\right\|_{p}$, входящие в $(3)$, упорядоченные в порядке убывания. Заметим, что из (3) следует соотношение

$$
\alpha_{i}(f, l) \ll i^{-1 / \theta} 2^{-l r_{1}} S_{l}
$$

Теперь из суммы $\sum_{l \leqslant(s, \gamma)<l+1} \delta_{s}(f, x)$ выберем те $m_{l}$ "блоков" $\delta_{s}(f, x)$, которьм соответствуют первые $m_{l}$ чисел $\alpha_{i}(f, l)$. Проделав эту процедуру для каждого $l \in\left[n, n_{0}\right)$, $l \in \mathbb{N}$, получим набор “блоков" $\delta_{s}(f, x)$, которые образуют некоторый полином. Этот полином мы и обозначим $R(x)$.

Нетрудно убедиться, что в таком случае количество гармоник $L$, содержащихся в $S_{M}(f, x)$, не превьшает по порядку $M$.

Действительно, в силу соотношения

$$
\sum_{(s, \gamma)<n} 2^{(s, 1)} \ll 2^{n} n^{\nu-1}
$$


(см. $\left[1\right.$, c. 11]) и выбора чисел $m_{l}$ будем иметь

$$
\begin{aligned}
L & \ll 2^{n} n^{\nu-1}+\sum_{l=n}^{n_{0}} 2^{l} m_{l} \ll 2^{n} n^{\nu-1}+2^{n} n^{\nu-1} \sum_{l=n}^{n_{0}} \sum_{l \leqslant(s, \gamma)<l+1} 2^{(s, r) \theta}\left\|\delta_{s}(f, x)\right\|_{p}^{\theta} \\
& \ll 2^{n} n^{\nu-1}+2^{n} n^{\nu-1}\|f\|_{B_{p, \theta}^{r}}^{\theta} \ll 2^{n} n^{\nu-1} \asymp M .
\end{aligned}
$$

Осталось убедиться, что полином $S_{M}(f, x)$ доставляет требуемую оценку приближения для $f(x) \in B_{p, \theta}^{r}$.

Пусть $D_{f}$ обозначает множество тех векторов $s: n \leqslant(s, \gamma)<n_{0}$, для которых "блоки" $\delta_{s}(f, x)$ не попали в $R(x)$. Тогда для $f(x) \in B_{p, \theta}^{r}$ будем иметь

$$
\begin{aligned}
\left\|f(x)-S_{M}(f, x)\right\|_{q} & =\left\|f(x)-\sum_{(s, \gamma)<n_{0}} \delta_{s}(f, x)+\sum_{s \in D_{f}} \delta_{s}(f, x)\right\|_{q} \\
& \leqslant\left\|f(x)-\sum_{(s, \gamma)<n_{0}} \delta_{s}(f, x)\right\|_{q}+\left\|\sum_{s \in D_{f}} \delta_{s}(f, x)\right\|_{q}=\mathscr{J}_{1}+\mathscr{J}_{2} .
\end{aligned}
$$

Согласно теореме 2 из [6] и выбору числа $n_{0}$ имеем

$$
\begin{aligned}
\mathscr{J}_{1} \ll 2^{-n_{0}\left(r_{1}-1 / p+1 / q\right)} & \leqslant 2^{-n\left(r_{1}-1 / p+1 / q\right)} n^{-(\nu-1)\left(r_{1}-1 / p+1 / q\right)} \\
& \asymp M^{-\left(r_{1}-1 / p+1 / q\right)} .
\end{aligned}
$$

Для оценки $\mathscr{J}_{2}$ воспользуемся соотношением $[1$, с. 25$]$

$$
\|f\|_{q} \ll\left\{\sum_{s}\left(\left\|\delta_{s}(f, x)\right\|_{p} 2^{\|s\|_{1}(1 / p-1 / q)}\right)^{q}\right\}^{1 / q}, \quad 1<p<q<\infty, \quad \text { и } \quad f(x) \in L_{q}\left(\pi_{d}\right) .
$$

В силу выбора чисел $\alpha_{i}(f, l)$ и их оценок, а также оценки $(7)$ получим

$$
\begin{aligned}
\mathscr{J}_{2} & \ll\left\{\sum_{l=n}^{n_{0}} \sum_{i>m_{l}} \alpha_{i}^{q}(f, l) 2^{l(1 / p-1 / q) q}\right\}^{1 / q} \\
& =\left\{\sum_{l=n}^{n_{0}} \sum_{i>m_{l}} \alpha_{i}^{\theta}(f, l) \alpha_{i}^{q-\theta}(f, l) 2^{l(1 / p-1 / q) q}\right\}^{1 / q} \\
& \ll\left\{\sum_{l=n}^{n_{0}} \sum_{i>m_{l}} \alpha_{i}^{\theta}(f, l) i^{-(q-\theta) / \theta} 2^{-l(q-\theta) r_{1}} S_{l}^{q-\theta} 2^{l(1 / p-1 / q) q}\right\}^{1 / q} \\
& \leqslant\left\{\sum_{l=n}^{n_{0}} m_{l}^{-(q-\theta) / \theta} 2^{-l(q-\theta) r_{1}} S_{l}^{q-\theta} 2^{l(1 / p-1 / q) q} \sum_{i>m_{l}} \alpha_{i}^{\theta}(f, l)\right\}^{1 / q} \\
& \ll\left\{\sum_{l=n}^{n_{0}} m_{l}^{-(q-\theta) / \theta} 2^{-l(q-\theta) r_{1}} S_{l}^{q-\theta} 2^{l(1 / p-1 / q) q} \sum_{l \leqslant(s, \gamma)<l+1}\left\|\delta_{s}(f, x)\right\|_{p}^{\theta}\right\}^{1 / q}
\end{aligned}
$$




$$
\begin{aligned}
& \ll\left\{\sum_{l=n}^{n_{0}} m_{l}^{-(q-\theta) / \theta} 2^{-l(q-\theta) r_{1}} S_{l}^{q-\theta} 2^{l(1 / p-1 / q) q} 2^{-l \theta r_{1}}\right. \\
& \left.\times \sum_{l \leqslant(s, \gamma)<l+1} 2^{(s, r) \theta}\left\|\delta_{s}(f, x)\right\|_{p}^{\theta}\right\}^{1 / q} \\
& \ll\left\{\sum_{l=n}^{n_{0}} m_{l}^{-(q-\theta) / \theta} 2^{-l\left(r_{1}-1 / p+1 / q\right) q} S_{l}^{q-\theta} S_{l}^{\theta}\right\}^{1 / q} \\
& =\left\{\sum_{l=n}^{n_{0}} m_{l}^{-(q-\theta) / \theta} 2^{-l\left(r_{1}-1 / p+1 / q\right) q} S_{l}^{q}\right\}^{1 / q} .
\end{aligned}
$$

Теперь, подставив в (8) вместо $m_{l}$ их соответствующие значения, продолжим оценку:

$$
\begin{aligned}
& \ll\left(2^{n} n^{\nu-1}\right)^{1 / q-1 / \theta}\left(\sum_{l=n}^{n_{0}} 2^{l(q-\theta) / \theta} S_{l}^{\theta-q} 2^{-l\left(r_{1}-1 / p+1 / q\right) q} S_{l}^{q}\right)^{1 / q} \\
& =\left(2^{n} n^{\nu-1}\right)^{1 / q-1 / \theta}\left(\sum_{l=n}^{n_{0}} S_{l}^{\theta} 2^{-l\left(r_{1}-1 / p+1 / q\right) q} 2^{l q(1 / \theta-1 / q)}\right)^{1 / q} \\
& =\left(2^{n} n^{\nu-1}\right)^{1 / q-1 / \theta}\left(\sum_{l=n}^{n_{0}} 2^{-l q\left(r_{1}-1 / p+2 / q-1 / \theta\right)} S_{l}^{\theta}\right)^{1 / q}=\left(2^{n} n^{\nu-1}\right)^{1 / q-1 / \theta} \mathscr{J}_{3} .
\end{aligned}
$$

Для оценки $\mathscr{J}_{3}$ рассмотрим два случая:

a) $r_{1} \geqslant 1 / p-2 / q+1 / \theta$

б) $1 / p-1 / q<r_{1}<1 / p-2 / q+1 / \theta$.

Итак, пусть имеет место случай а). Тогда

$$
\begin{aligned}
\mathscr{J}_{3} & \leqslant 2^{-n\left(r_{1}-1 / p+2 / q-1 / \theta\right)}\left(\sum_{l=n}^{n_{0}} S_{l}^{\theta}\right)^{1 / q} \\
& =2^{-n\left(r_{1}-1 / p+2 / q-1 / \theta\right)}\left(\sum_{l=n}^{n_{0}} \sum_{l \leqslant(s, \gamma)<l+1}\left\|\delta_{s}(f, x)\right\|_{p}^{\theta} 2^{(s, r) \theta}\right)^{1 / q} \\
& \ll 2^{-n\left(r_{1}-1 / p+2 / q-1 / \theta\right)}\|f\|_{B_{p, \theta}^{r}}^{\theta / q} \leqslant 2^{-n\left(r_{1}-1 / p+2 / q-1 / \theta\right)} .
\end{aligned}
$$

Сопоставив (8)-(10), приходим к оценке

$$
\begin{aligned}
\mathscr{J}_{2} & \ll\left(2^{n} n^{\nu-1}\right)^{1 / q-1 / \theta} 2^{-n\left(r_{1}-1 / p+2 / q-1 / \theta\right)}=2^{-n\left(r_{1}-1 / p+1 / q\right)} n^{(\nu-1)(1 / q-1 / \theta)} \\
& \asymp M^{-\left(r_{1}-1 / p+1 / q\right)}\left(\log ^{\nu-1} M\right)^{r_{1}-1 / p+2 / q-1 / \theta} .
\end{aligned}
$$

Пусть теперь $1 / p-1 / q<r_{1}<1 / p-2 / q+1 / \theta$. В таком случае

$$
\mathscr{J}_{3} \leqslant 2^{-n_{0}\left(r_{1}-1 / p+2 / q-1 / \theta\right)}\left(\sum_{l=n}^{n_{0}} S_{l}^{\theta}\right)^{1 / q}
$$




$$
\begin{aligned}
& =2^{-n_{0}\left(r_{1}-1 / p+2 / q-1 / \theta\right)}\left(\sum_{l=n}^{n_{0}} \sum_{l \leqslant(s, \gamma)<l+1} 2^{(s, r) \theta}\left\|\delta_{s}(f, x)\right\|_{p}^{\theta}\right)^{1 / q} \\
& \leqslant 2^{-n_{0}\left(r_{1}-1 / p+2 / q-1 / \theta\right)}\|f\|_{B_{p, \theta}^{r}}^{\theta / q} \\
& \leqslant 2^{-n\left(r_{1}-1 / p+2 / q-1 / \theta\right)} n^{-(\nu-1)\left(r_{1}-1 / p+2 / q-1 / \theta\right)}
\end{aligned}
$$

и согласно (8), (9) и (12) получаем оценку

$$
\begin{aligned}
\mathscr{J}_{2} & \ll\left(2^{n} n^{\nu-1}\right)^{1 / q-1 / \theta} 2^{-n\left(r_{1}-1 / p+2 / q-1 / \theta\right)} n^{-(\nu-1)\left(r_{1}-1 / p+2 / q-1 / \theta\right)} \\
& =2^{-n\left(r_{1}-1 / p+1 / q\right)} n^{-(\nu-1)\left(r_{1}-1 / p+1 / q\right)} \asymp M^{-\left(r_{1}-1 / p+1 / q\right)} .
\end{aligned}
$$

Наконец, подставляя поочередно оценки (11) и (6), а затем (13) и (6) в (5), приходим к искомой оценке сверху величины $e_{M}^{\perp}\left(B_{p, \theta}^{r}\right)_{q}$ в случае $1 \leqslant \theta<q$. Оценка сверху в теореме установлена.

Переходя к доказательству в (2) оценки снизу, обратим внимание, что ее достаточно получить при $\nu=d$.

Пусть $f(x) \in B_{p, \theta}^{r}$ и $S_{M}(f, x)$ - полином, составленный из $M$ гармоник ции $f(x)$, выбранных произвольньм образом. Тогда в силу следствия Д1.2 (см. [7, с. 392])

$$
\left\|f(x)-S_{M}(f, x)\right\|_{q}=\sup _{g(x):\|g\|_{q^{\prime}} \leqslant 1}\left|\int_{\pi_{d}}\left(f(x)-S_{M}(f, x)\right) g(x) d x\right|,
$$

где $q^{-1}+\left(q^{\prime}\right)^{-1}=1$.

Подберем число $l \in \mathbb{N}$ таким образом, чтобы вьполнялись соотношения $2^{l} l^{d-1} \asymp M$ и $2^{l} l^{d-1} \geqslant 2 M$, и рассмотрим функцию

$$
F(x)=\sum_{(s, 1)=l} \sum_{k \in \rho(s)} e^{i(k, x) \stackrel{\mathrm{df}}{=}} \sum_{(s, 1)=l} d_{s}(x) .
$$

Поскольку

$$
\left\|\delta_{s}(F, x)\right\|_{p}=\left\|d_{s}(x)\right\|_{p} \asymp 2^{(s, 1)(1-1 / p)}, \quad 1<p<\infty,
$$

то из (15) согласно теореме о характеризации класса $H_{p}^{r}=B_{p, \infty}^{r}($ см. $[1$, с. 32$])$ заключаем, что функция

$$
f_{1}(x)=C_{1} 2^{-l\left(r_{1}+1-1 / p\right)} F(x)
$$

с соответствующей постоянной $C_{1}>0$ принадлежит классу $B_{p, \infty}^{r}$.

Аналогично, если $1 \leqslant \theta<\infty$, то из определения классов $B_{p, \theta}^{r}$ и с учетом (15) будем иметь

$$
\begin{aligned}
\|F\|_{B_{p, \theta}^{r}} & =\left(\sum_{(s, 1)=l} 2^{(s, r) \theta}\left\|\delta_{s}(F, x)\right\|_{p}^{\theta}\right)^{1 / \theta}=\left(\sum_{(s, 1)=l} 2^{(s, r) \theta}\left\|\delta_{s}\left(d_{s}, x\right)\right\|_{p}^{\theta}\right)^{1 / \theta} \\
& \asymp\left(\sum_{(s, 1)=l} 2^{(s, r) \theta} 2^{(s, 1)(1-1 / p) \theta}\right)^{1 / \theta}=\left(\sum_{(s, 1)=l} 2^{(s, 1)\left(r_{1}+1-1 / p\right) \theta}\right)^{1 / \theta} \\
& \ll 2^{l\left(r_{1}+1-1 / p\right)} l^{(d-1) / \theta} .
\end{aligned}
$$


Следовательно, функция

$$
f_{2}(x)=C_{2} 2^{-l\left(r_{1}+1-1 / p\right)} l^{-(d-1) / \theta} F(x), \quad C_{2}>0,
$$

принадлежит классу $B_{p, \theta}^{r}, 1 \leqslant \theta<\infty$.

Функцию $g(x)$, которая фигурирует в $(14)$, подберем, также отправляясь от функции $F(x)$.

Поскольку (см. [8]) $\|F\|_{q^{\prime}} \ll 2^{l / q} l^{(d-1) / q^{\prime}}, 1 \leqslant q^{\prime}<\infty$, то легко видеть, что функция

$$
g(x)=C_{3} 2^{-l / q} l^{-(d-1) / q^{\prime}} F(x), \quad C_{3}>0,
$$

при соответствующем выборе постоянной $C_{3}$ удовлетворяет неравенству $\|g\|_{q^{\prime}} \leqslant 1$.

Пусть $1 \leqslant \theta<\infty$. Тогда, подставив построенные функции (17) и (18) в (14) и принимая во внимание, что $M \asymp 2^{l} l^{d-1}$, получаем

$$
\begin{aligned}
\left\|f_{2}(x)-S_{M}\left(f_{2}, x\right)\right\|_{q} & \gg\left(2^{l} l^{d-1}-M\right) 2^{-l\left(r_{1}+1-1 / p+1 / q\right)} l^{-(d-1)\left(1 / \theta+1 / q^{\prime}\right)} \\
& \gg 2^{l} l^{d-1} 2^{-l\left(r_{1}+1-1 / p+1 / q\right)} l^{-(d-1)\left(1 / \theta+1 / q^{\prime}\right)} \\
& =2^{-l\left(r_{1}-1 / p+1 / q\right)} l^{(d-1)(1 / q-1 / \theta)} \\
& \asymp M^{-\left(r_{1}-1 / p+1 / q\right)}\left(\log ^{d-1} M\right)^{r_{1}-1 / p+2 / q-1 / \theta} .
\end{aligned}
$$

Если же $\theta=\infty$, то, подставив в (14) функции (16) и (18) и проведя соответствующие преобразования, получим оценку

$$
\left\|f_{1}(x)-S_{M}\left(f_{1}, x\right)\right\|_{q} \gg M^{-\left(r_{1}-1 / p+1 / q\right)}\left(\log ^{d-1} M\right)^{r_{1}-1 / p+2 / q} .
$$

В завершение доказательства обратим внимание, что оценки (19) при $r_{1} \geqslant 1 / p-$ $2 / q+1 / \theta$ и $(20)$ при $r_{1}>1 / p-1 / q$ совпадают по порядку с соответствующими оценками сверху для $e_{M}^{\perp}\left(B_{p, \theta}^{r}\right)_{q}$. Что касается случая $1 / p-1 / q<r_{1}<1 / p-2 / q+1 / \theta$, то здесь в силу независимости оценки сверху величины $e_{M}^{\perp}\left(B_{p, \theta}^{r}\right)_{q}$ от размерности пространства $\mathbb{R}^{d}$ ее соответствующую оценку снизу достаточно получить в случае $d=1$. Но, с другой стороны, оценка (19) в одномерном случае точна для всех $r_{1}>1 / p-1 / q$. Теорема доказана.

ЗАмЕчаниЕ 1. Сопоставив результат доказанной теоремы с результатом теоремы 2 из [6], обнаруживаем, что в случае $1 / p-1 / q<r_{1}<1 / p-2 / q+1 / \theta$ величины $e_{M}^{\perp}\left(B_{p, \theta}^{r}\right)_{q}$ убывают при $M \rightarrow \infty$ быстрее верхних граней уклонений ступенчатых гиперболических сумм $Ф$ уье, содержащих по порядку $M$ гармоник. На классах $H_{p}^{r}$, как следует из теоремы 1 при $\theta=\infty$ и соответствующего результата из [1, теорема 2.2, c. 35$]$, подобного рода эффект места не имеет.

Теперь перейдем к исследованию величин $e_{M}^{\perp}\left(B_{p, \theta}^{r}\right)_{q}$ для других соотношений между параметрами $p$ и $q$.

Имеют место следуюшие утверждения.

Теорема 2. Пусть $1<q \leqslant p<\infty, p \geqslant 2, r_{1}>0$. Тогда прu $1 \leqslant \theta \leqslant \infty$ справедлива оченка

$$
e_{M}^{\perp}\left(B_{p, \theta}^{r}\right)_{q} \asymp M^{-r_{1}}\left(\log ^{\nu-1} M\right)^{\left(r_{1}+1 / 2-1 / \theta\right)_{+}} .
$$


TeOpema 3. Пусть $1<q<p \leqslant 2$. Тогда

а) если $1 \leqslant \theta<p, r_{1} \geqslant 1 / \theta-1 / p$ или $\theta \geqslant p u r_{1}>0$, mo

$$
M^{-r_{1}}\left(\log ^{\nu-1} M\right)^{r_{1}+1 / 2-1 / \theta} \ll e_{M}^{\perp}\left(B_{p, \theta}^{r}\right)_{q} \ll M^{-r_{1}}\left(\log ^{\nu-1} M\right)^{r_{1}+1 / p-1 / \theta} ;
$$

б) если $1 \leqslant \theta<p u 0<r_{1}<1 / \theta-1 / p$, mo

$$
e_{M}^{\perp}\left(B_{p, \theta}^{r}\right)_{q} \asymp M^{-r_{1}}
$$

Перед тем как перейти к доказательству сформулированных теорем, отметим следующее. Поскольку при $p \geqslant 2 B_{p, \theta}^{r} \subset B_{2, \theta}^{r}$, то, с одной стороны, оценка сверху в теореме 2 является следствием оценки сверху в теореме 3 при $p=2$. С другой стороны, поскольку при $1<p \leqslant 2 B_{2, \theta}^{r} \subset B_{p, \theta}^{r}$, то оценки снизу в теореме 3 получим как следствие оценки снизу в теореме 2 .

ДОКАЗАТЕЛЬСТВО ТЕОРЕМ 2 и 3 . В первую очередь отметим, что оценка сверху в теореме 3 при $p \leqslant \theta \leqslant \infty$ следует из соответствующей оценки приближения класса $B_{p, \theta}^{r}$ ступенчатыми гиперболическими суммами Фурье (см. [9]). Таким образом, получим оценку сверху величины $e_{M}^{\perp}\left(B_{p, \theta}^{r}\right)_{q}$ при $1<q<p \leqslant 2$ и $1 \leqslant \theta<p$. При этом мы будем проводить рассуждения, аналогичные тем, что и при установлении оценки сверху в теореме 1. Не останавливаясь на деталях этих рассуждений, отметим только некоторые их отличительные моменты.

Для $f(x) \in B_{p, \theta}^{r}$ приближающий полином будем выбирать в виде

$$
S_{M}(f, x)=\sum_{\left(s, \gamma^{\prime}\right)<n} \delta_{s}(f, x)+R_{1}(x),
$$

где $R_{1}(x)$ строится по известной схеме с тем отличием, что вместо величины $S_{l}$ при его построении используется величина

$$
\widetilde{S}_{l}=\left(\sum_{l \leqslant\left(s, \gamma^{\prime}\right)<l+1} 2^{(s, r) \theta}\left\|\delta_{s}(f, x)\right\|_{p}^{\theta}\right)^{1 / \theta},
$$

т.е. в суммировании вектор $\gamma$ заменяется на $\gamma^{\prime}$.

Итак, предположим, что полином $S_{M}(f, x)$ построен. Получим оценку сверху для $\left\|f(x)-S_{M}(f, x)\right\|_{q}, f(x) \in B_{p, \theta}^{r}$.

Пусть $\widetilde{D}_{f}$ обозначает множество тех векторов $s: n \leqslant\left(s, \gamma^{\prime}\right)<n_{0}$, для которых "блоки" $\delta_{s}(f, x)$ не попали в $R_{1}(x)$. Тогда

$$
\begin{aligned}
\left\|f(x)-S_{M}(f, x)\right\|_{q} & \leqslant\left\|f(x)-\sum_{\left(s, \gamma^{\prime}\right)<n_{0}} \delta_{s}(f, x)+\sum_{s \in \widetilde{D}_{f}} \delta_{s}(f, x)\right\|_{q} \\
& \leqslant\left\|f(x)-\sum_{\left(s, \gamma^{\prime}\right)<n_{0}} \delta_{s}(f, x)\right\|_{q}+\left\|\sum_{s \in \widetilde{D}_{f}} \delta_{s}(f, x)\right\|_{q}=\mathscr{J}_{4}+\mathscr{J}_{5} .
\end{aligned}
$$


Согласно оценке приближения класса $B_{p, \theta}^{r}$ ступенчатыми гиперболическими суммами Фурье (см. [9]) имеем

$$
\mathscr{J}_{4} \ll 2^{-n_{0} r_{1}} \asymp M^{-r_{1}} .
$$

Чтобы оценить слагаемое $\mathscr{J}_{5}$, воспользуемся сначала теоремой Литтлвуда-Пэли (см., например, [2, с. 59-65]) и затем неравенством $|a+b|^{c} \leqslant|a|^{c}+|b|^{c}, 0 \leqslant c \leqslant 1$. В результате получим

$$
\begin{aligned}
\mathscr{J}_{5} & =\left\|\sum_{s \in \widetilde{D}_{f}} \delta_{s}(f, x)\right\|_{q} \leqslant\left\|\sum_{s \in \widetilde{D}_{f}} \delta_{s}(f, x)\right\|_{p} \\
& \ll\left\|\left(\sum_{s \in \widetilde{D}_{f}}\left|\delta_{s}(f, x)\right|^{2}\right)^{1 / 2}\right\|_{p} \leqslant\left(\sum_{s \in \widetilde{D}_{f}}\left\|\delta_{s}(f, x)\right\|_{p}^{p}\right)^{1 / p} .
\end{aligned}
$$

Далее, повторив рассуждения, которые проводились при оценке $\mathscr{J}_{2}$ (см. (8), (9)), из (23) получаем

$$
\mathscr{J}_{5} \ll\left(2^{n} n^{\nu-1}\right)^{1 / p-1 / \theta}\left(\sum_{l=n}^{n_{0}} 2^{-p l\left(r_{1}-1 / \theta+1 / p\right)} \widetilde{S}_{l}^{\theta}\right)^{1 / p} .
$$

Чтобы продолжить оценку (24), рассмотрим два случая:

a) $r_{1} \geqslant 1 / \theta-1 / p$

б) $0<r_{1}<1 / \theta-1 / p$.

Итак, пусть $r_{1} \geqslant 1 / \theta-1 / p$. Тогда

$$
\begin{aligned}
\mathscr{J}_{5} & \ll\left(2^{n} n^{\nu-1}\right)^{1 / p-1 / \theta} 2^{-n\left(r_{1}-1 / \theta+1 / p\right)}\left(\sum_{l=n}^{n_{0}} \widetilde{S}_{l}^{\theta}\right)^{1 / p} \\
& \ll 2^{-n r_{1}} n^{(\nu-1)(1 / p-1 / \theta)}\|f\|_{B_{p, \theta}^{r}}^{\theta / p} \ll 2^{-n r_{1}} n^{(\nu-1)(1 / p-1 / \theta)} \\
& \asymp M^{-r_{1}}\left(\log ^{\nu-1} M\right)^{r_{1}+1 / p-1 / \theta} .
\end{aligned}
$$

Подставив $(25)$ и $(22)$ в $(21)$, приходим к оценке

$$
\left\|f(x)-S_{M}(f, x)\right\|_{q} \ll M^{-r_{1}}\left(\log ^{\nu-1} M\right)^{r_{1}+1 / p-1 / \theta} .
$$

Пусть теперь $0<r_{1}<1 / \theta-1 / p$. Тогда из $(24)$ находим

$$
\begin{aligned}
\mathscr{J}_{5} & \ll\left(2^{n} n^{\nu-1}\right)^{1 / p-1 / \theta} 2^{-n_{0}\left(r_{1}-1 / \theta+1 / p\right)}\left(\sum_{l=n}^{n_{0}} \widetilde{S}_{l}^{\theta}\right)^{1 / p} \\
& \ll\left(2^{n} n^{\nu-1}\right)^{1 / p-1 / \theta} 2^{-n\left(r_{1}-1 / \theta+1 / p\right)} n^{-(\nu-1)\left(r_{1}-1 / \theta+1 / p\right)} \\
& =2^{-n r_{1}} n^{-(\nu-1) r_{1}} \asymp M^{-r_{1}} .
\end{aligned}
$$

Таким образом, оценки сверху в теореме 3 , а следовательно, и в теореме 2 установлены. 
Переходя к оценке снизу в теореме 2 , заметим, что ее достаточно получить в случае $1<q \leqslant 2<p<\infty$ и при $\nu=d$. Аналогично доказательству оценки снизу в теореме 1 здесь мы также будем использовать соотношение (14).

Подберем число $l$ из условий $2^{l} l^{d-1} \asymp M$ и $2^{l} l^{d-1} \geqslant 2 M$ и положим

$$
F_{1}(x)=\sum_{(s, 1)=l} \prod_{j=1}^{d} R_{s_{j}}\left(x_{j}\right)
$$

где $R_{m}(t)=\sum_{k=2^{m-1}}^{2^{m}-1} \varepsilon_{k} e^{i k t}, \varepsilon_{k}= \pm 1,-$ полиномы Рудина-Шапиро (см., например, [10, c. 155]), обладающие свойством $\left\|R_{m}\right\|_{\infty} \ll 2^{m / 2}$.

Итак, пусть $1 \leqslant \theta<\infty$. Тогда

$$
\begin{aligned}
\left\|F_{1}\right\|_{B_{p, \theta}^{r}} & \asymp\left(\sum_{(s, 1)=l} 2^{(s, r) \theta}\left\|\delta_{s}\left(F_{1}, x\right)\right\|_{p}^{\theta}\right)^{1 / \theta} \\
& \ll\left(\sum_{(s, 1)=l} 2^{(s, r) \theta} 2^{(s, 1 / 2) \theta}\right)^{1 / \theta}=\left(\sum_{(s, 1)=l} 2^{(s, 1)\left(r_{1}+1 / 2\right) \theta}\right)^{1 / \theta} \\
& =2^{l\left(r_{1}+1 / 2\right)}\left(\sum_{(s, 1)=l} 1\right)^{1 / \theta} \asymp 2^{l\left(r_{1}+1 / 2\right)} l^{(d-1) / \theta} .
\end{aligned}
$$

Отсюда заключаем, что функция

$$
f_{3}(x)=C_{4} 2^{-l\left(r_{1}+1 / 2\right)} l^{-(d-1) / \theta} F_{1}(x)
$$

с соответствующей постоянной $C_{4}>0$ принадлежит классу $B_{p, \theta}^{r}, 1 \leqslant \theta<\infty$. Аналогично из определения класса $B_{p, \infty}^{r}$ и свойства полиномов Рудина-Шапиро следует, что функция

$$
f_{4}(x)=C_{5} 2^{-l\left(r_{1}+1 / 2\right)} F_{1}(x), \quad C_{5}>0,
$$

принадлежит классу $B_{p, \infty}^{r}$.

Функцию $g(x)$, которая фигурирует в (14), также подберем, отправляясь от функции $F_{1}(x)$. Поскольку при $2 \leqslant q^{\prime}<\infty$

$$
\begin{aligned}
\left\|F_{1}\right\|_{q^{\prime}} & \ll\left\|\left(\sum_{(s, 1)=l}\left|\delta_{s}\left(F_{1}, x\right)\right|^{2}\right)^{1 / 2}\right\| \|_{q^{\prime}} \leqslant\left(\sum_{(s, 1)=l}\left\|\delta_{s}\left(F_{1}, x\right)\right\|_{q^{\prime}}^{2}\right)^{1 / 2} \\
& \ll 2^{l / 2}\left(\sum_{(s, 1)=l} 1\right)^{1 / 2} \asymp 2^{l / 2} l^{(d-1) / 2}
\end{aligned}
$$

отсюда следует, что функция

$$
g_{1}(x)=C_{6} 2^{-l / 2} l^{-(d-1) / 2} F_{1}(x), \quad C_{6}>0,
$$


удовлетворяет условию $\left\|g_{1}\right\|_{q^{\prime}} \leqslant 1$. Подставив $f_{3}(x)$ и $g_{1}(x)$ в $(14)$, будем иметь

$$
\begin{aligned}
\left\|f_{3}(x)-S_{M}\left(f_{3}, x\right)\right\|_{q} & \gg\left(2^{l} l^{d-1}-M\right) 2^{-l\left(r_{1}+1\right)} l^{-(d-1)(1 / 2+1 / \theta)} \\
& \gg 2^{l} l^{d-1} \cdot 2^{-l\left(r_{1}+1\right)} l^{-(d-1)(1 / 2+1 / \theta)}=2^{-l r_{1}} l^{(d-1)(1 / 2-1 / \theta)} \\
& \asymp M^{-r_{1}}\left(\log ^{d-1} M\right)^{r_{1}+1 / 2-1 / \theta}
\end{aligned}
$$

Соответственно, подставив в $(14) f_{4}(x)$ и $g_{1}(x)$, приходим к оценке

$$
\left\|f_{4}(x)-S_{M}\left(f_{4}, x\right)\right\|_{q} \gg M^{-r_{1}}\left(\log ^{d-1} M\right)^{r_{1}+1 / 2} .
$$

Оценки (27) и (28) совпадают по порядку с соответствующими оценками сверху в теореме 2 при $r_{1} \geqslant 1 / \theta-1 / 2$. Если же $0<r_{1}<1 / \theta-1 / 2$, то искомые оценки снизу следуют из одномерного случая. Оценки снизу в теореме 2 и, следовательно, в теореме 3 установлены. Теоремы 2 и 3 доказаны.

ЗАмЕЧАниЕ 2. Сравнив оценки теорем 2 и 3 с соответствующими оценками приближения классов $B_{p, \theta}^{r}$ ступенчатыми гиперболическими суммами Фурье (см. [6] и [9]), обнаруживаем (при $0<r_{1}<1 / \theta-1 / 2$ и $0<r_{1}<1 / \theta-1 / p$ соответственно) их различие в смысле порядка. На классах $H_{p}^{r}$ отмеченный эффект места не имеет.

Обратим внимание, что в теореме 3 в отличие от теоремы 2 не охвачен случай $p=q$. В этом случае нам удалось установить точные по порядку оценки величин $e_{M}^{\perp}\left(B_{p, \theta}^{r}\right)_{p}$, $p \in(1,2)$.

Имеет место

Teоpema 4. Пусть $p \in(1,2), r_{1}>0$. Тогда $n p u 1 \leqslant \theta \leqslant \infty$

$$
e_{M}^{\perp}\left(B_{p, \theta}^{r}\right)_{p} \asymp M^{-r_{1}}\left(\log ^{\nu-1} M\right)^{\left(r_{1}+1 / p-1 / \theta\right)_{+}} .
$$

ДокАЗАТЕЛЬСтво. Оценка сверху в (29) устанавливается с помощью тех же рассуждений, что и в теореме 3 . Мы не будем на этом останавливаться, а только отметим, что для оценки слагаемого $\mathscr{J}_{4}$ нужно воспользоваться результатом теоремы 1 [6].

Установим оценку снизу. При этом мы будем следовать схеме рассуждений, которая применялась В.Н. Темляковым в $[1$, с. 94$]$ при получении им оценки снизу величины наилучшего $M$-членного тригонометрического приближения класса $W_{p, \alpha}^{r}$ в пространстве $L_{q}$ при $1<p \leqslant q \leqslant 2$.

По числу $M$ подберем $n$ таким, чтобы $M \asymp 2^{n} n^{d-1}$ и число точек во множестве $F_{n}=\bigcup_{(s, 1)=n} \rho(s)$ было бы больше $4 M$. Рассмотрим функции

$$
f_{5}(x)=C_{7} 2^{-n\left(r_{1}+1-1 / p\right)} n^{-(d-1) / \theta} \sum_{k \in F_{n}} e^{i(k, x)}, \quad C_{7}>0,
$$

при $1 \leqslant \theta<\infty$ и

$$
f_{6}(x)=C_{8} 2^{-n\left(r_{1}+1-1 / p\right)} \sum_{k \in F_{n}} e^{i(k, x)}, \quad C_{8}>0,
$$

при $\theta=\infty$, которые, как было показано при доказательстве теоремы 1 , принадлежат классам $B_{p, \theta}^{r}, 1 \leqslant \theta<\infty$, и $B_{p, \infty}^{r}$ соответственно. 
Далее, пусть $\Omega_{M}$ - произвольный набор из $M$ целочисленных векторов $k^{1}, \ldots, k^{M}$, $k^{j}=\left(k_{1}^{j}, \ldots, k_{d}^{j}\right)$. Для каждого вектора $s:(s, 1)=n$ рассмотрим множества $\Omega_{M} \cap \rho(s)$. Нетрудно видеть, что согласно выбору числа $n$ множество тех векторов $s:(s, 1)=n$, для которых $\left|\Omega_{M} \cap \rho(s)\right| \leqslant \frac{1}{2}|\rho(s)|$, будет содержать, по крайней мере, половину всех векторов $s$, удовлетворяющих условию $(s, 1)=n$. Поэтому, обозначив множество этих векторов через $S$, можем записать $|S| \asymp n^{d-1}$. Далее нам понадобится вспомогательное утверждение (см. [1, с. 28]).

Лемма 1. Пусть $1<p<q \leqslant \infty u f(x) \in L_{p}\left(\pi_{d}\right)$. Тогда

$$
\|f\|_{p} \gg\left(\sum_{s}\left\|\delta_{s}(f, x)\right\|_{q}^{p} 2^{\|s\|_{1}(1 / q-1 / p) p}\right)^{1 / p} .
$$

Итак, пусть $S_{M}\left(f_{5}, x\right)$ - полином, составленньй из гармоник Фурье функции $f_{5}(x)$, имеюших "номера" в $\Omega_{M}$. Тогда согласно (30) для $f_{5}(x)$ будем иметь оценку

$$
\begin{aligned}
\left\|f_{5}(x)-S_{M}\left(f_{5}, x\right)\right\|_{p} & \gg\left(\sum_{(s, 1)=n}\left\|\delta_{s}\left(f_{5}(x)-S_{M}\left(f_{5}, x\right)\right)\right\|_{2}^{p} 2^{\|s\|_{1}(1 / 2-1 / p) p}\right)^{1 / p} \\
& \gg\left(\sum_{s \in S}\left\|\delta_{s}\left(f_{5}(x)-S_{M}\left(f_{5}, x\right)\right)\right\|_{2}^{p}\right)^{1 / p} 2^{n(1 / 2-1 / p)} \\
& \gg 2^{-n\left(r_{1}+1-1 / p\right)} n^{-(d-1) / \theta}|S|^{1 / p} 2^{n(1 / 2-1 / p)} 2^{n / 2} \\
& \asymp 2^{-n r_{1}} n^{(d-1)(1 / p-1 / \theta)} \asymp M^{-r_{1}}\left(\log ^{d-1} M\right)^{r_{1}+1 / p-1 / \theta} .
\end{aligned}
$$

Заметим, что полученная оценка совпадает по порядку с оценкой сверху величины $e_{M}^{\perp}\left(B_{p, \theta}^{r}\right)_{p}$ в случае $r_{1} \geqslant 1 / \theta-1 / p$. Если же $0<r_{1}<1 / \theta-1 / p$, то искомая оценка снизу следует из одномерного случая. Аналогично, для $f_{6}(x)$ согласно $(31)$ получаем

$$
\left\|f_{6}(x)-S_{M}\left(f_{6}, x\right)\right\|_{p} \gg 2^{-n r_{1}} n^{(d-1) / p} \asymp M^{-r_{1}}\left(\log ^{d-1} M\right)^{r_{1}+1 / p} .
$$

Теорема доказана.

ЗАмЕЧАниЕ 3 . Сопоставив результат теоремы $4 \mathrm{c}$ результатом теоремы 1 [6], видим, что при $0<r_{1}<1 / \theta-1 / p$ величины $e_{M}^{\perp}\left(B_{p, \theta}^{r}\right)_{p}$ убывают при $M \rightarrow \infty$ быстрее верхних граней уклонений ступенчатых гиперболических сумм Фурье.

В заключение приведем два результата о приближении классов $W_{p, \alpha}^{r}$.

Teоpema 5. Пусть $1<p \leqslant q<\infty, r_{1}>1 / p-1 / q$. Тогда

$$
e_{M}^{\perp}\left(W_{p, \alpha}^{r}\right)_{q} \asymp M^{-\left(r_{1}-1 / p+1 / q\right)}\left(\log ^{\nu-1} M\right)^{\left(r_{1}-2 / p+2 / q\right)_{+}} .
$$

ДокаЗАТЕЛЬСтво. В первую очередь отметим, что оценка (32) в случаях $1<p \leqslant$ $2<q<\infty$ и $1<p \leqslant q \leqslant 2$ известна [5]. Поэтому рассмотрим случай $2 \leqslant p<q<\infty$. Оценка сверху здесь следует из теоремы 1 согласно вложению $W_{p, \alpha}^{r} \subset B_{p, p}^{r}, 2 \leqslant p<\infty$, а соответствующая оценка снизу устанавливается с помощью рассуждений, проведенных при доказательстве теоремы 1 . При этом в роли функции $f_{1}(x)$ нужно выбрать функцию

$$
f_{7}(x)=C_{9} 2^{-l\left(r_{1}-1 / p+1\right)} l^{-(d-1) / p} F(x), \quad C_{9}>0,
$$

которая, как нетрудно проверить, принадлежит классу $W_{p, \alpha}^{r}$. Теорема доказана. 
ЗАмЕчАниЕ 4. Сопоставив (32) с результатом теоремы $2.1[1$, с. 34$]$, видим, что при $1 / p-1 / q<r_{1} \leqslant 2(1 / p-1 / q)$ имеет место различие в поведении величин $e_{M}^{\perp}\left(W_{p, \alpha}^{r}\right)_{q}$ по сравнению с величинами верхних граней уклонений ступенчатых гиперболических сумм

СлЕДСТВИЕ 1. Пусть $1<q<p<\infty, r_{1}>0$. Тогда

$$
e_{M}^{\perp}\left(W_{p, \alpha}^{r}\right)_{q} \asymp M^{-r_{1}}\left(\log ^{\nu-1} M\right)^{r_{1}}
$$

Оценка сверху в (33) следует из оценки приближения класса $W_{p, \alpha}^{r}$ ступенчатыми гиперболическими суммами Фурье в метрике $L_{p}, 1<p<\infty$ (см. [1, теорема 2.1]). Оценка снизу является следствием оценки снизу $M$-членных тригонометрических приближений (см. следствие 1 в [2]).

Таким образом, порядки величин $e_{M}^{\perp}\left(W_{p, \alpha}^{r}\right)_{q}$ в следствии 1 совпадают с порядками верхних граней уклонений ступенчатых гиперболических сумм Фурье, а также наилучших $M$-членных тригонометрических приближений (см., например, [2]).

ЗАмечание 5. Обнаруженные вьше эффекты на классах $B_{p, \theta}^{r}$ и $W_{p, \alpha}^{r}$ имеют многомерную специфику и в отмеченных случаях в пространстве $\mathbb{R}^{1}$ не проявляются.

\section{СПИСОК ЦИТИРОВАННОЙ ЛИТЕРАТУРЫ}

[1] Темляков В.Н. Приближение функций с ограниченной смешанной производной // Тр. МИАН. 1986. Т. 178.

[2] Кашин Б. С., Темляков В. Н. О наилучших $m$-членных приближениях и энтропии множеств в пространстве $L_{1} / /$ Матем. заметки. 1994. Т. 56. № 5. С. 57-86.

[3] Лизоркин П.И., Никольский С. М. Пространства функций смешанной гладкости с декомпозиционной точки зрения // Тр. МИАН. 1989. Т. 187. С. 143-161.

[4] Никольский С. М. Приближение функций многих переменных и теоремы вложения. М.: Наука, 1969.

[5] Белинский Э.С. Приближение плавающей системой экспонент на классах периодических функций с ограниченной смешанной производной // Исследования по теории функций многих вещественных переменных. Ярославль: ЯГУ, 1988. С. 16-33.

[6] Романюк А. С. Приближение классов Бесова периодических функций многих переменных в пространстве $L_{q} / /$ Укр. матем. ж. 1991. Т. 43. № 10. С. 1398-1408.

[7] Корнейчук Н.П. Точные константы в теории приближения. М.: Наука, 1987.

[8] Галеев Э. М. Порядковые оценки производных периодического многомерного $\alpha$-ядра Дирихле в смешанной норме // Матем. сб. 1982. Т. 117. № 1. С. 32-43.

[9] Галеев Э. М. Приближение классов периодических функций нескольких переменных ядерными операторами // Матем. заметки. 1990. Т. 47. № 3. С. 32-41.

[10] Кашин Б. С., Саакян А. А. Ортогональные ряды. М.: Наука, 1984.

Институт математики НАН Украины, г. Киев

Поступило

E-mail: funct@imath.kiev.ua

16.03 .2000 